\title{
Increased Radiosensitivity of Granulocyte Macrophage Colony-forming Units and Skin Fibroblasts in Human Autosomal Recessive Severe Combined Immunodeficiency
}

\author{
M. Cavazzana-Calvo, F. Le Deist, G. De Saint Basile, D. Papadopoulo, ^ J. P. De Villartay, and A. Fischer \\ Institut National de la Santé et de la Recherche Médicale U 132, Hôpital Necker-Enfants Malades, 149, rue de Sèvres, \\ 75015 Paris, France; and *Institut Curie, Section de Biologie, 26, rue d'Ulm, 75231 Paris Cedex 05, France
}

\begin{abstract}
We studied the radiosensitivity of granulocyte macrophage colony-forming units (GM-CFU) in patients with a severe combined immunodeficiency (SCID). Three patients lacking both mature $T$ and $B$ cells showed a twofold higher GM-CFU radiosensitivity calculated as the DO value (dose required to reduce survival to $37 \%$ ), and an identical observation was made with fibroblasts from one of these patients. A patient with an SCID with hypereosinophilia, i.e., Omenn's syndrome characterized by extremely restricted $T$ cell heterogeneity and a lack of $B$ cells, also showed abnormal GM-CFU radiosensitivity. In contrast, GM-CFU from a patient lacking only $T$ cells ( $X$-linked form of SCID) showed normal GM-CFU radiosensitivity. These data further support the similarity between human $\mathrm{T}(-)$ B (-) SCID and the murine scid mutation characterized by a defect in $\mathbf{T}$ cell receptor and immunoglobulin gene rearrangement, and by an abnormal double-strand DNA break repair function. In addition, they strongly suggest that the Omenn's immunodeficiency syndrome may be a leaky $\mathrm{T}(-) \mathrm{B}(-)$ SCID phenotype as previously indicated by the coexistence of the two phenotypes in siblings. (J. Clin. Invest. 1993. 91:1214-1218.) Key words: human severe combined immunodeficiency $\bullet$ DNA repair • radiosensitivity • granulocyte macrophage colony-forming units • skin fibroblast
\end{abstract}

\section{Introduction}

The murine severe combined immunodeficiency (SCID) ${ }^{1} \mathrm{mu}-$ tation leads to faulty $T$ and $B$ cell differentiation because of impaired coding joint formation during both immunoglobulin and $T$ cell receptor $V(D) J$ gene rearrangements $(1-3)$. The gene involved has been assigned to chromosome $16(4)$ but not

Address correspondence to Marina Cavazzana-Calvo, INSERM U 132, Hôpitai Necker-Enfants Malades, 149, rue de Sévres, 75730 Paris Cedex 15, France.

Received for publication 10 July 1992 and in revised form 15 October 1992.

1. Abbreviations used in this paper: GM-CFU, granulocyte macrophage colony-forming units; SCID, severe combined immunodeficiency.

J. Clin. Invest.

(c) The American Society for Clinical Investigation, Inc. 0021-9738/93/03/1214/05 \$2.00

Volume 91, March 1993, 1214-1218 characterized. The higher radiosensitivity of granulocyte macrophage colony-forming units (GM-CFU) and fibroblasts of scid mice appears result from defective double-strand DNA break repair after irradiation (5-7). An equivalent immunological phenotype of adenosine deaminase $(+)$ SCID in humans is characterized by the absence of mature $T$ and $B$ lymphocytes, while natural killer cells are present (8). This disease shows autosomal recessive inheritance. Recently, Schwarz et al. (9) provided evidence for an abnormal recombination pattern of $D_{H}$ to $J_{H}$ elements in pre-B cells from such patients, leading to the suggestion that the human disease may resemble the murine form.

We analyzed the radiosensitivity of marrow GM-CFU from three patients with the $\mathrm{T}(-) \mathrm{B}(-)$ SCID phenotype, healthy controls, and a patient with another type of SCID: X-linked $\mathrm{T}(-) \mathrm{B}(+)(10)$. We also tested GM-CFU from a patient with the Omenn immunodeficiency syndrome, a condition that could correspond to a leaky scid phenotype (11) as in scid mice (12). GM-CFU from $\mathrm{T}(-) \mathrm{B}(-)$ patients and patient with Omenn's showed increased radiosensitivity, comparable to that of murine scid GM-CFU, while GM-CFU from the patient with the X-linked $\mathrm{T}(-) \mathrm{B}(+)$ SCID phenotype showed normal radiosensitivity. Skin fibroblasts from one $\mathrm{T}(-) \mathrm{B}(-)$ SCID patient also exhibited increased radiosensitivity.

\section{Methods}

Patients. SCID was diagnosed according to the World Health Organization definition (8). An absence of antigen-specific, cell-mediated (in vitro $\mathrm{T}$ cell proliferation and skin tests), and humoral immune responses was the main common feature of the five patients studied ( Table I). Adenosine deaminase and purine nucleoside phosphorylase activities were normal, as was major histocompatibility complex class I and class II antigen expression. Lymphocyte subsets were characterized by using immunofluorescence techniques (13), with the following monoclonal antibodies: Leu4 (anti-CD3 IgG2a; Becton Dickinson, Mountain View, CA), Leu3a (anti-CD4 IgG1; Becton Dickinson), Leu2a (anti-CD8 IgG; Becton Dickinson), Leu5b (anti-CD2 IgG2a; Becton Dickinson), Leul1b (anti-CD16 IgG1; Becton Dickinson), anti-HLA class II IgG2a (Becton Dickinson), anti-CD25: rIL2 (IgG1; Becton Dickinson), and antimembrane IgM (Nordic Immunology, Tilburg, The Netherlands).

Patient 5 had a high blood $\mathrm{T}$ cell count, as well as $\mathrm{T}$ cell infiltration of skin, gut, and marrow. T cells expressed MHC class II molecules (46\%) but not CD25. A restricted heterogeneity of T lymphocytes rearrangement, previously found to be characteristic of Omenn's syndrome, was detected as reported for unrelated patients (11). Discrete non-germline band rearrangement of the $\beta 1, \beta 2$, and $\gamma$ chain genes 
Table I. Clinical and Immunological Characterization of Severe Combined Immunodeficiency Patients

\begin{tabular}{lrrrrr}
\hline & 1 & 2 & 3 & 4 & 5 \\
\hline Sex & Male & Male & Male & Male & Male \\
Age & 6 mo & 6 mo & 5 mo & 6 mo & 4 mo \\
Lymphocytes $/ \mathrm{mm}^{3}$ & 200 & 500 & 900 & 1,500 & 9,600 \\
CD2* & 85 & ND & 76 & 52 & ND \\
CD3* & 6 & 0 & 0 & 26 & 88 \\
CD4 $^{*}$ & 8 & 2 & 5 & 2 & 55 \\
CD8* & 97 & 53 & 28 & 39 & 36 \\
mIgM* $_{\text {CD16* }}^{*}$ & 0 & 0 & 0 & 46 & 0 \\
Maternal T cells & 78 & 68 & 11 & ND & ND \\
& $(+)$ & $(-)$ & $(-)$ & $(+)$ & $(-)$ \\
& & & & Caryotyping &
\end{tabular}

* Results are expressed as the percentage of positive lymphocytes. ND, not done, mIgM, membrane IgM.

were observed using the $C \beta 1$ (14) and $\mathrm{J} \gamma 1$ (15) probes on EcoRI- and HindIII-digested T cell DNA (Fig. 1, $A$ and $B$, arrows).

Maternal $T$ cell engraftment was proven by caryotyping in patient 4. No maternal $T$ cells were found by HLA typing in patients 2,3 , and 5. To test the possible $X$-linked inheritance of the $B^{+}$SCID in patient 4 , $\mathrm{X}$-chromosome inactivation was studied in leukocytes from the mother since there was no family history of SCID. X-chromosome inactivation analysis was performed by methylation-sensitive enzymatic digestion of DNA isolated from PMN, T lymphocytes, or pooled EBV-derived maternal B cell lines using the M27 $\beta$ (16) probe as previously described (17). Both T and B lymphocytes but not PMN from the mother exhibited a skewed pattern of X-chromosome inactivation (data not shown) typical of obligate XL-SCID carriers (18).

Marrow cell isolation. Mononuclear cells from the patients and healthy marrow donors were isolated by density gradient centrifugation over Ficoll-Hypaque ( $d=1.077$; Pharmacia, Uppsala, Sweden ). Since the marrow of patient 5 was infiltrated by $T$ cells, the latter were depleted by rosetting with 2-aminoethylisothiouronium bromidetreated red cells. A control marrow sample was similarly treated. Informed consent was obtained from all the patients' parents. Origin of patients 1 and 2 bone marrow cells was controlled by variable number tandem repeat amplification analysis indicating host origin.

Skin fibroblast cell strains and culture conditions. Two skin fibroblast cell lines were used. The human skin fibroblast cell line $1 \mathrm{BR} / 3$ supplied by Dr. C. Arlett (University of Sussex, United Kingdom) and a skin fibroblast cell line derived from a $\mathrm{T}(-) \mathrm{B}(-)$ SCID patient (patient 2 in Table I) $(19,20)$. The cells were routinely cultured in MEM (Gibco Laboratories, Grand Island, NY) supplemented with glutamine $(2 \mathrm{mM})$, gentamycin $(40 \mu \mathrm{g} / \mathrm{ml})$, and $15 \%$ fetal calf serum (Gibco Laboratories). Cultures were maintained in humidified 7\% $\mathrm{CO}_{2}$ atmosphere at $37^{\circ} \mathrm{C}$. In all experiments, the cells used were in exponential growth phase.

Irradiation. Marrow mononuclear cells were suspended in RPMI 1640 (Gibco Laboratories) at a density of $1 \times 10^{6} / \mathrm{ml}$ in polypropylene tubes (Falcon Labware, Oxnard, CA). Irradiation was delivered at 1 $\mathrm{Gy} / \mathrm{min}$ by $\mathrm{a}^{137} \mathrm{Cs}$ irradiator for various times. Skin fibroblast cell lines in exponential growth phase were irradiated by a ${ }^{137} \mathrm{Cs}$ irradiator at dose rate $0.45 \mathrm{~Gy} / \mathrm{min}$.

Granulocyte macrophage progenitor cell colony assay (GM-CFU). Human GM-CFU were assayed as previously described (21). Briefly, cells suspended in MEM (Gibco Laboratories) containing $0.8 \%$ methylcellulose, $30 \%$ fetal calf serum (Gibco Laboratories), 10\% BSA (Sigma Immunochemicals, St. Louis, MO), $2 \mathrm{mM}$ glutamine, and 0.1 $\mathrm{mM}$ mercaptoethanol were plated in duplicate at $1 \times 10^{5}$ cells $/ 35-\mathrm{mm}$ petri dishes. Culture supernatant from the 5637 cell line (22), a source
A

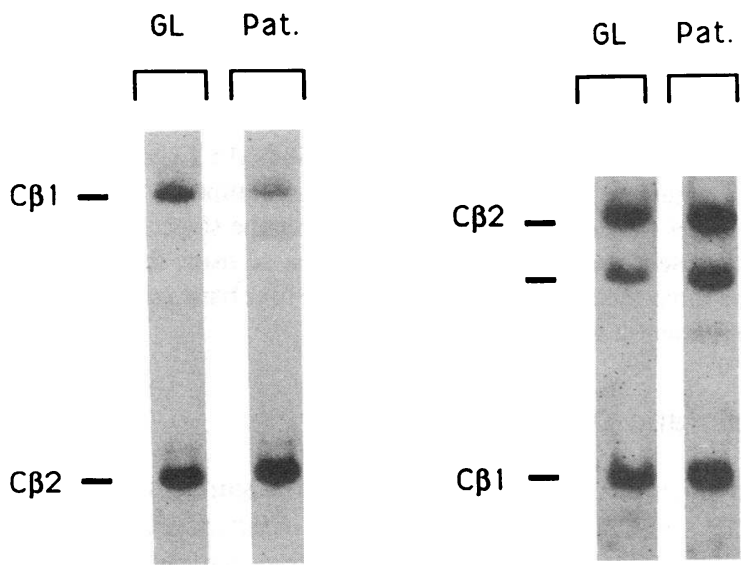

B

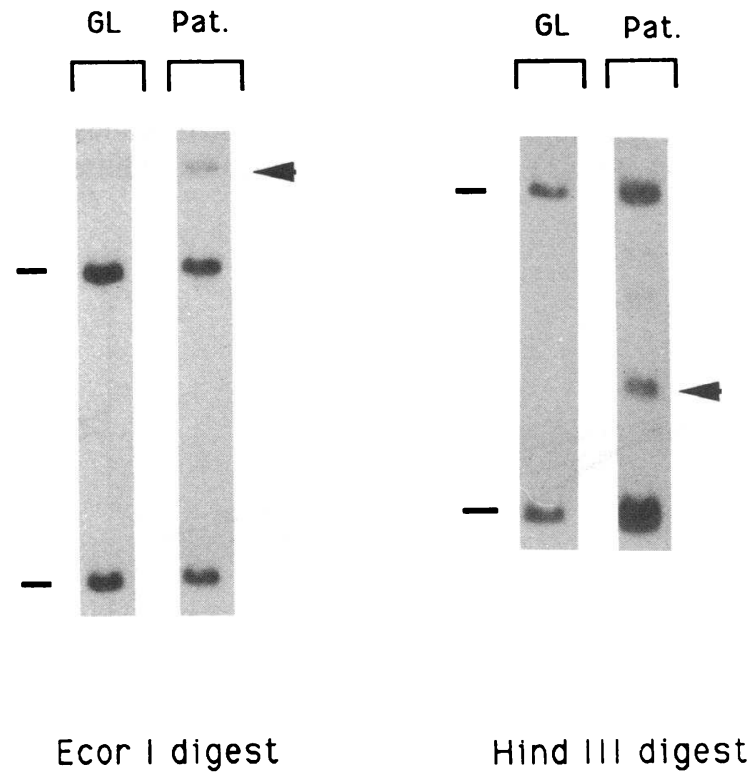

Figure 1. Southern hybridization of $\mathrm{C} \beta 1(A)$ and $\mathrm{J} \gamma 1(\mathrm{pH} 6.0)(B)$ probes to EcoRI- and HindIII-digested DNA from T cells of patient 4 (Pat.) and nonlymphoid cells $(G L)$. - Germline band; $\rightarrow$ nongermline band. Two additional faint non-germline line bands (not indicated by arrows) can be seen with the HindIII digest $(B)$.

of colony stimulating factor, was used at a final concentration of $10 \%$. Colonies were scored by examination with an inverted microscope after incubation at $37^{\circ} \mathrm{C}$ in a humidified atmosphere of $5 \% \mathrm{CO}_{2}$-air for $14 \mathrm{~d}$. Plates with colonies containing more than 50 cells were scored as positive and the survival determined as the percent colony-forming cells compared to an unirradiated control. Radiation survival curves were plotted by least-squares linear regression analysis. Results were expressed as DO values, i.e., the dose of radiation required to reduce survival to $37 \%$ in the exponential section of the survival curve and the extrapolation number that is the surviving fraction obtained by extrapolating the survival curve to zero dose (23).

Skin fibroblast cell survival. Immediately after treatment, the cells were washed with PBS, trypsinized, suspended in complete medium, and counted using a ZM Coulter Counter (Coulter Electronics, Margency, France). An appropriate number of cells was seeded in plastic 
dishes (diameter $100 \mathrm{~mm}$ ) (Falcon Labware). For each dose of irradiation 9-12 dishes were plated. The plates were incubated for $16 \mathrm{~d}$, after which the cells fixed with methanol and stained with $10 \%$ Giemsa solution. The cloning efficiency of untreated cells ranged from 15 to $22 \%$ for $1 \mathrm{BR} / 3,5$ to $12 \%$ for the cell line from $\mathrm{T}(-) \mathrm{B}(-)$ SCID patient. Survival was calculated as percentage of the cloning efficiency of untreated cultures.

Statistical analysis. To estimate changes in GM-CFU radiation sensitivity in marrow samples of each patient as compared to controls a linear model was used (log scale). Thereafter the slopes of the linear models (patient versus controls) were compared using the Student's $t$ test. The same method was used to compare fibroblast radiation sensitivity of patient 2 to normal controls.

\section{Results and Discussion}

As shown in Fig. 2, GM-CFU in marrow samples from three $\mathbf{T}(-) \mathbf{B}(-)$ SCID patients exhibited a clear increase in radiosensitivity $(P<0.001)$, corresponding to a twofold decrease in DO values ( Table II ). It was not possible to repeat these experiments because patients' marrow samples could obviously not be obtained repeatedly. It is, however, noteworthy that the three samples showed the same increase GM-CFU radiosensitivity as in the case of GM-CFU and fibroblasts from scid mice (5). In addition, the skin fibroblast of SCID patient 2 was also studied (Fig. 3) and showed an increased radiosensitivity ( $P$
$<0.001)$. In comparison with the skin fibroblasts $(1 \mathrm{BR} / 3)$ derived from a normal donor, fibroblasts from patient 2 were threefold more sensitive according to DO value ( $1.75 \mathrm{~Gy}$ and $0.55 \mathrm{~Gy}$, respectively). Reported normal values of fibroblasts DO vary between 1.0 and $1.7 \mathrm{~Gy}(19)$. These data strongly suggest that the human $\mathrm{T}(-) \mathrm{B}(-)$ SCID phenotype may also correspond to a defect in double-strand DNA break repair (7) leading to impaired recombinase activity in T and B cells (5). Indeed, Schwartz et al. (9) have recently provided evidence for defective $D_{H}$ to $J_{H}$ rearrangements in marrow pre-B cells from $\mathbf{T}(-) \mathrm{B}(-)$ SCID patients with extensive nucleotide deletions at the DQ52-J junction, while no such deletion in the D-J immunoglobulin coding elements were found in pre-B cells from $\mathbf{T}(-) \mathrm{B}(+)$ SCID patients. This concords with the observation that GM-CFU from a $\mathrm{T}(-) \mathrm{B}(+)$ SCID patient exhibited a normal radiosensitivity pattern (patient 4 , Fig. 3 ). The latter finding also showed that abnormal GM-CFU radiosensitivity did not result from the absence of marrow $T$ cells.

The SCID mutation in the mouse is often accompanied by a variable degree of leakiness, defined by the presence of a small number of $\mathrm{T}$ and/or B cell clones (3). We have previously proposed that the so-called Omenn's syndrome, which is characterized by skin and gut $\mathrm{T}$ cell infiltration and a profound immunodeficiency (11), could be a "leaky" phenotype of the human $\mathrm{T}(-) \mathrm{B}(-) \operatorname{SCID}(12)$. This was based $(a)$ on the very

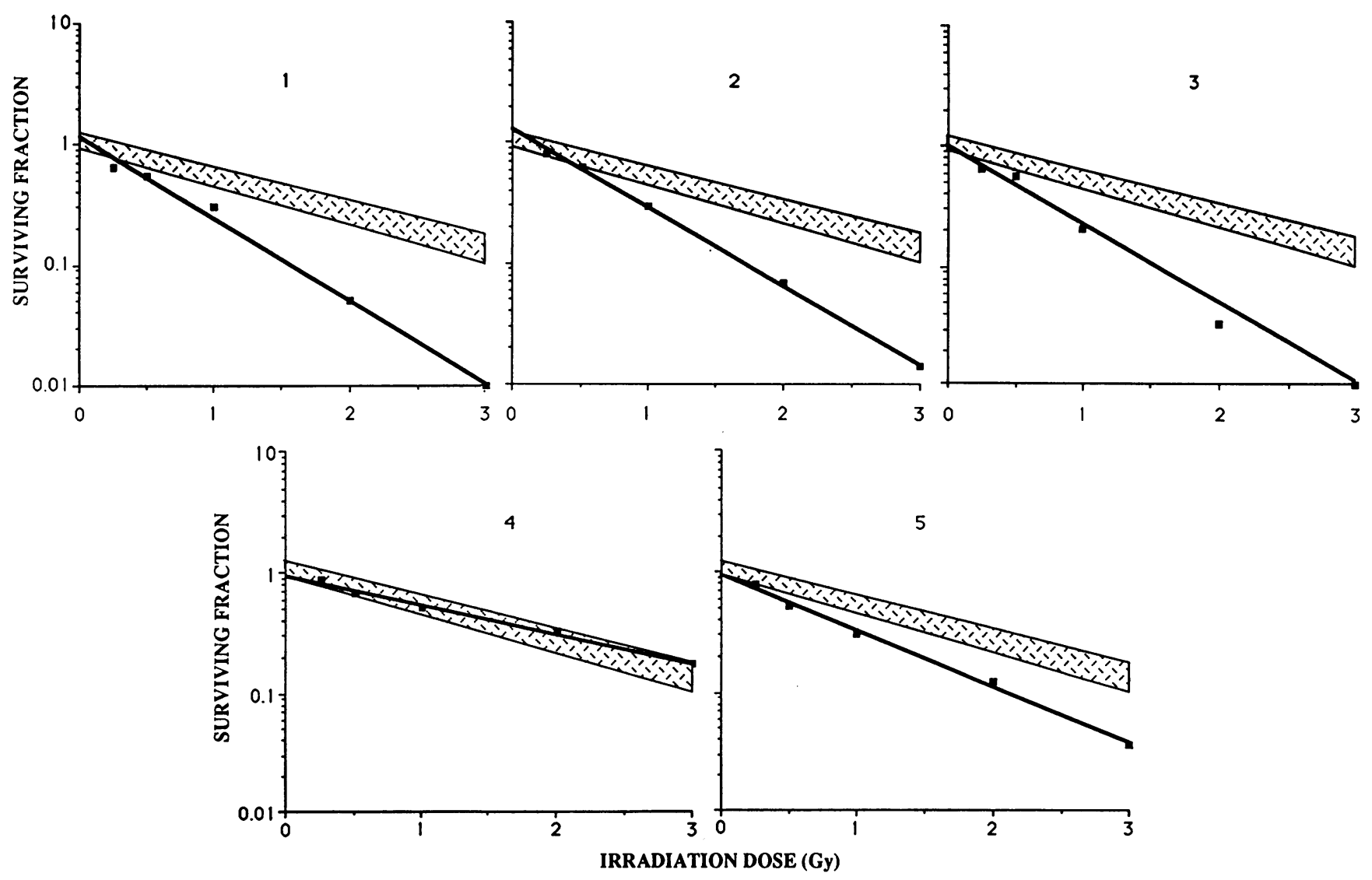

Figure 2. Survival curves of GM-CFU from bone marrow of healthy subjects and SCID patients. The number of surviving GM-CFU were measured by plotting the cells in methylcellulose with the appropriate growth factors as described previously (see Methods). The number of GMCFU in the unirradiated suspension was defined as 1; the number of GM-CFU in the unirradiated suspensions was expressed as a fraction of the corresponding unirradiated controls. Shaded area corresponds to mean \pm 1 SD of GM-CFU radiosensitivity of control bone marrow cells. (1-3) T(-) B(-) SCID; (4) XL-linked T(-) B(+) SCID; (5) Omenn's syndrome. Survival curves of GM-CFU of patients 1, 2, 3, and 5 were statistically different from that of control bone marrow cells $(P<0.001)$. 
Table II. Summary of Survival Curves for GM-CFU of Healthy Control, $T^{-} B^{-} S C I D, T^{-} B^{+} S C I D$, and Omenn's Syndrome

\begin{tabular}{lcccc}
\hline & & & \multicolumn{2}{c}{ Mean values \pm SD } \\
\cline { 3 - 5 } Normal control & DO $(G y)$ & $n$ value & DO & $n$ value \\
\hline 1 & 1.915 & 1.12 & $1.541 \pm 0.23$ & $1.038 \pm 0.05$ \\
2 & 1.447 & 1.02 & & \\
3 & 1.456 & 0.98 & & \\
4 & 1.305 & 1.03 & & \\
5 & 1.583 & 1.04 & & \\
$\mathrm{~T}^{-} \mathrm{B}^{-} \mathrm{SCID}$ & & & & \\
1 & 0.83 & 1.28 & $0.74 \pm 0.09$ & $1.16 \pm 0.11$ \\
2 & 0.73 & 1.16 & & \\
3 & 0.65 & 1.05 & & \\
$\mathrm{~T}^{-} \mathrm{B}^{+}$SCID & & & & \\
1 & 1.69 & 0.95 & & \\
Ommen's syndrome & & & & \\
1 & 0.83 & 0.88 & & \\
& & & &
\end{tabular}

Each entry indicates results of a survival curve measurement made on cells from a single marrow sample. To determine the survival curves for GM-CFU bone marrow was exposed to various doses of $\gamma$-radiation from $\mathrm{a}^{137} \mathrm{Cs}$ irradiator. The cells were cultured as described in the text. DO is the radiation dose required to reduce the surviving fraction of cells to 0.37 in the exponential portion of the survival curve; $n$, the extrapolation number, is the surviving fraction obtained when the linear portion of the curve is extrapolated to zero dose and the intersection with the log survival axis is read as $n$.

restricted heterogeneity of T cell receptor $\alpha \beta$ and/or $\gamma \delta$ usage in four cases studied and $(b)$ on a case of concomitant $\mathrm{T}(-)$ $\mathrm{B}(-)$ SCID and Omenn's syndrome in two siblings. This hypothesis is supported by the present observation that the radiosensitivity of GM-CFU from a patient with a typical Omenn's syndrome (and TCR-restricted heterogeneity) (Fig. 1) was sim-

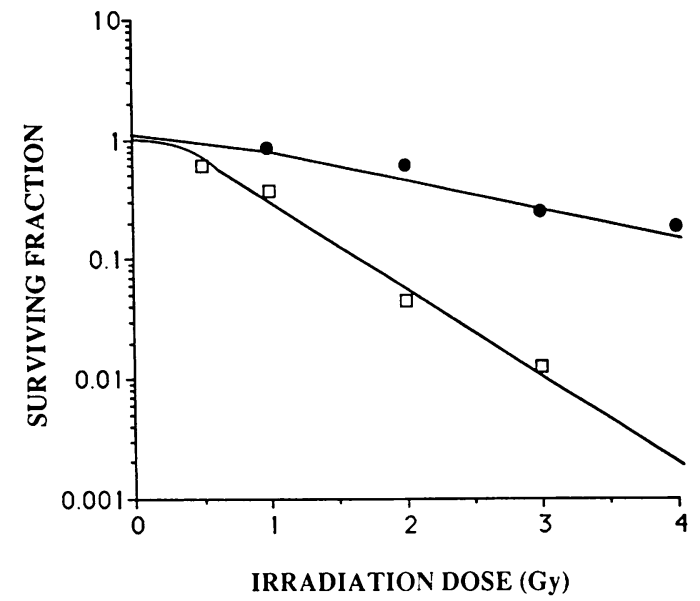

Figure 3. Survival curves of skin fibroblasts from a normal subject (1BR3 - - -) and patient 2 (T(-) B(-) SCID - - -). The survival fraction was expressed as a fraction of the corresponding unirradiated controls. The mean values of two experiments are shown. Survival parameters were for $1 \mathrm{BR} / 3: \mathrm{DO}=1.75 \mathrm{~Gy}$ and $\mathrm{Dq}=0.9$ and for the patient 2: $\mathrm{DO}=0.55$ and $\mathrm{Dq}=0.35$. Survival curve of skin fibroblast from patient 2 was statistically different from that of normal control $(P<0.001)$. ilar to that of GM-CFU from T(-) B(-) SCID patients (patient 5, Fig. 2). Human $\mathrm{T}(-) \mathrm{B}(-)$ SCID may thus correspond, like ataxia telangiectasia (24), to a disease caused by a gene anomaly with pleiotropic effects on DNA, i.e., antigen receptor rearrangement and DNA repair defects. No clinical consequences of the increase radiosensitivity have yet been described in $\mathrm{T}(-) \mathrm{B}(-)$ SCID patients, possibly because of the early death of nontransplanted patients and to the fact that irradiation is not part of the conditioning regimen before bone marrow transplantation therapy. Further studies are now required to determine whether in human $\mathrm{T}(-) \mathrm{B}(-)$ SCID the increased GM-CFU and possibly fibroblast radiosensitivity is due to a defect in double-strand DNA break repair like in mice (7). Murine scid gene has been assigned to chromosome 16 proximal to mouse immunoglobulin $\lambda$ light chain gene ( Igl-1) (4). It will be interesting to assess whether the human $T(-)$ $\mathrm{B}(-)$ SCID locus maps to the syntenic region.

In conclusion, there are now three elements indicating that the human $\mathrm{T}(-) \mathrm{B}(-)$ SCID phenotype is closely related to the mouse scid mutation, i.e., abnormal $D_{H}$ to $J_{H}$ rearrangement (9), increased GM-CFU, and possibly fibroblast radiosensitivity and leakiness $(11,12)$. The parallel finding in murine and human SCID of abnormal antigen receptor recombination and increased radiosensitivity strongly suggests that both defects are the consequence of a single mutation affecting a gene encoding a molecule implicated in DNA repair. Since the number of tested patients is limited, it is still possible that there might be distinct subsets of $\mathrm{T}(-) \mathrm{B}(-)$ SCID.

\section{Acknowledgments}

We are grateful to Ms. C. Fromont for the technical assistance, to Ms. D. Bresson for typing the manuscript, as well as to Dr. H. Magdelenat (Institut Curie, Paris, France) for precious advice in the study of radiosensitivity of normal bone marrow cells. We thank Drs. M. P. Lefranc, T. Rabbitts, and T. W. Mak for the generous gift of $\beta$ and $\delta$ T cell receptor probe. We gratefully acknowledge the help of Dr. Sylvie Chevret, Département de Biostatistique et Informatique Médicale, Hôpital Saint-Louis, Paris, for careful analysis of experimental data.

This work was supported by Institut National de la Santé et de la Recherche Médicale.

\section{References}

1. Bosma, G. C., R. P. Custer, and M. J. Bosma. 1983. A severe combined immunodeficiency mutation in the mouse. Nature (Lond.). 301:527-530.

2. Carroll, A. M., and H. J. Bosma. 1991. T-lymphocyte development in SCID mice is arrested shortly after the initiation of T-cell receptor gene recombination. Genes \& Dev. 5:1357-1366.

3. Phillips, R. A., and D. E. Spaner. 1991. The scid mouse: mutation in a DNA repair gene creates recipients useful for studies on stem cells, lymphocyte development and graft-versus-host disease. Immunol. Rev. 124:63-74.

4. Bosma, G. C., M. T. Davisson, N. R. Ruetsch, H. O. Sweet, L. D. Schutz, and M. J. Bosma. 1989. The mouse mutation severe combined immune deficiency (scid) is on chromosome 16. Immunogenetics. 29:54-57.

5. Fulop, G. M., and R. A. Philipps. 1990. The scid mutation in mice causes a general defect in DNA repair. Nature (Lond.). 347:479-482.

6. Hendrickson, E. A., X. Q. Qin, E. A. Bump, D. G. Schatz, M. Oettinger, and D. T. Weaver. 1991. A link between double-strand break-related repair and V(D)J recombination: the scid mutation. Proc. Natl. Acad. Sci. USA. 88:40614065.

7. Biedermann, K. A., J. R. Sum, A. J. Giaccia, L. M. Tosto, and J. M. Brown. 1991. Scid mutation in mice confers hypersensitivity to ionizing radiation and a deficiency in DNA double-strand break repair. Proc. Natl. Acad. Sci. USA. 88:1394-1397.

8. Primary Immunodeficiency Diseases. Report of a World Health Organisation sponsored meeting. 1989. Immunodefic. Rev. 1:173. 
9. Schwarz, K., T. E. Hausen-Hagge, C. Knobloch, W. Friedrich, and E. Kleihauer. 1991. Severe combined immunodeficiency (SCID) in man: B cell-negative (B-) SCID patients exhibit an irregular recombination pattern at the $\mathbf{J}_{\mathrm{H}}$ locus. J. Exp. Med. 174:1039-1048.

10. Griscelli, C., A. Durandy, J. L. Virelizier, J. L. Ballet, and F. Daguillard 1978. Selective defect of precursor T cells associated with apparently normal B lymphocytes in severe combined immunodeficiency disease. J. Pediatr. 93:404411.

11. De Saint Basile, G., F. Le Deist, J. P. De Villartay, N. Cerf-Bensussan, O. Journet, N. Brousse, C. Griscelli, and A. Fischer. 1991. Restricted heterogeneity of $\mathrm{T}$ lymphocytes in combined immunodeficiency with hypereosinophilia $(\mathrm{Om}$ men's syndrome). J. Clin. Invest. 87:1352-1359.

12. Bosma, G. C., M. Fried, R. P. Custer, A. Carroll, D. M. Gibson, and M. J. Bosma. 1988. Evidence of functional lymphocytes in some (leaky) scid mice. $J$. Exp. Med. 167:1016-1033.

13. Le Deist, F., G. Thoenes, J. Corado, B. Lisowska-Grospierre, and A Fischer. 1991. Immunodeficiency with low expression of the T cell receptor/CD3 complex. Effect on T lymphocyte activation. Eur. J. Immunol. 21:1641-1648.

14. Yanagi, Y., A. Chan, B. Chiu, M. D. Minden, and T. W. Mak. 1985. Analysis of cDNA clones specific for human T cells and the $\beta$ chains of the T cell receptor heterodimer from human T cell line. Proc. Natl. Acad. Sci. USA. $82: 3430-3434$

15. Lefranc, M. P., and T. H. Rabbits. 1985. Two tandemly organized human genes encoding the T-cell constant region sequences show multiple rearrangement in different $T$ cell types. Nature (Lond.). 316:463-464.

16. Boyd, Y., and N. J. Fraser. 1990. Methylation patterns at the hypervari- able X-chromosome locus DXS255 (M27 $\beta$ ): correlation with X-inactivation status. Genomics. 7:182-187.

17. Arveiler, B., G. De Saint Basile, A. Fischer, C. Griscelli, and J. L. Mandel. 1990. Germ-line mosaicism simulates genetic heterogeneity in Wiskott-Aldrich syndrome. Am. J. Hum. Genet. 46:906-911.

18. Conley, M. E., A. Lavoie, Briggs, P. Brown, C. Guerra, and J. M. Puck. 1988. Nonrandom X-chromosome inactivation in B cells from carrier X-chromosome-linked severe combined immunodeficiency. Proc. Natl. Acad. Sci. USA. 85:3090-3094.

19. Arlett, C. F., S. A. Harcourt, A. R. Lehman, S. Stervens, and B. A. Bridges. 1975. Ataxia telangiectasia: a human mutation with abnormal radiation sensitivity. Nature (Lond.). 258:427-429.

20. Papadopoulo, D., D. Averbeck, and E. Moustachi. 1987. The fate of 8-methoxypsoralen photoinduced DNA interstrand crosslinks in Fanconi's anemia cells of defined genetic complementation groups. Mutat. Res. 184:271-280.

21. Coulombel, L., A. C. Eaves, and C. J. Eaves. 1983. Enzymatic treatment of long-term human marrow cultures reveals the preferential location of primitive hemopoietic progenitors in the adherent layer. Blood. 62:291-297.

22. Fogh, J. 1978. Cultivation, characterization and identification of human tumor cells with emphasis on kidney, testis and bladder tumors. Natl. Cancer Inst. Monogr. 49:5.

23. Senn, J. S., and E. A. McCulloch. 1970. Radiation sensitivity of human bone marrow cells measured by a cell culture method. Blood. 35:56-60.

24. Swift, M. 1990. Genetic aspects of ataxia-telangiectasia. Immunodefic. Rev. 2:67-81. 